\title{
AFM Applications in Petrochemical Polymers
}

Jiang Liu

ATOFINA Petrochemicals, Inc., Research and Technology Center, PO Box 1200, Deer Park, TX 77536.

DI AFM TappingMode was used in the industrial characterizations of polymers for product development and processing improvement at ATOFINA Petrochemicals, Inc. Some examples of the applications are presented here to show the value of this technology.

Surface roughness analysis of hazy films Surface roughness analysis of polypropylene (PP) (Fig 1) and polyethylene (PE) (Fig. 2) films has correlated strong relationships between surface roughness and level of haze for these thin films. It has been found that surface roughness dominates the level of haze for thinner films due to light scattering on the surfaces.

Crystallization introduced surface defects of thin films Crystallization in isotactic PP (iPP) thin film has been found being the source of "splotchiness" defects as the dendrites showing in Fig. 3. The corresponding phase image from the same area suggests these dendrites have changed the physical properties of the film surface. In some cases, these dendrites could be thicker "dashedlines" in the machine (drawing) direction (Fig. 3b). Spherulites on film surface were also seen lined up in the machine direction (Fig. 3c), increased the haze level of the thin films.

Phase dispersion of ethylene propylene rubber (EPR) in impact copolymers (ICP) Phase imaging has been successfully used in the characterization of EPR particle dispersion in impact copolymers, which provides convenient means for new product development and processing improvement. Fig. 4 shows the examples of images of polymer from a reactor (a) and from an extruder (b). Higher resolution image reveals the coexistence of three phases of PP, EPR and PE in the material. Image analysis based on these phase images provided information on particle size and size distribution.

Phase imaging for rubber dispersion in high impact polystyrene (HIPS) Phase imaging is also used in the characterization of polybutadiene rubber particle dispersion in polystyrene matrix for HIPS product development (Fig. 5). In addition to rubber particle size and size distribution, higher magnification images also provided detailed structure information of these particles. Cryomicrotoming and AFM imaging found cutting temperature is critical for obtaining smooth cutting faces of HIPS specimens from different formulations.

Phase dispersion in polymer blends Fig. 6 shows height (a) and phase (b) images, from the same area, of a slit tape of EPR and iPP blend. All rubber particles were seen elongated in the drawing direction and were forced out in some degree from the tape surface, which makes the slit tape surface rougher and therefore more opaque. Fig. 7 is a phase image of metallocene PE (mPE) and iPP blend, which provided visual information on the PE dispersion in the PP matrix.

AFM phase imaging has become an alternative analytical method of traditional TEM characterization for materials with different phases such as ICP, HIPS and polymer blends discussed above. AFM possesses advantages over TEM in terms of equipment and operational cost, sample preparation and image analysis. 

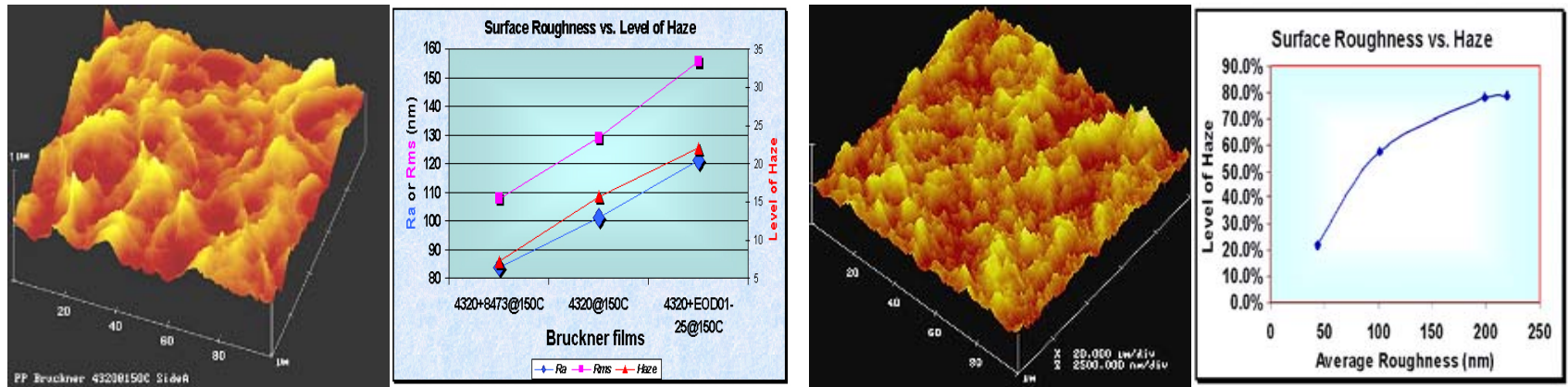

Fig.1. PP thin film surface roughness vs. haze.

Fig. 2. Thicker PE film surface roughness vs. haze.

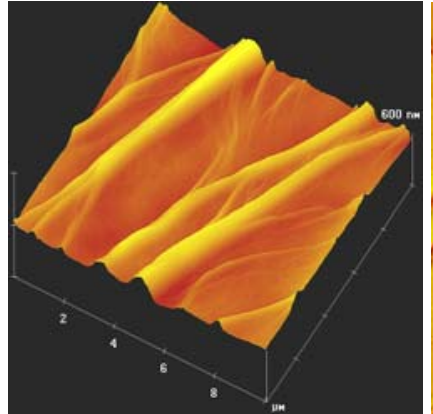

(a) Dendrite height (left) and phase images (right).

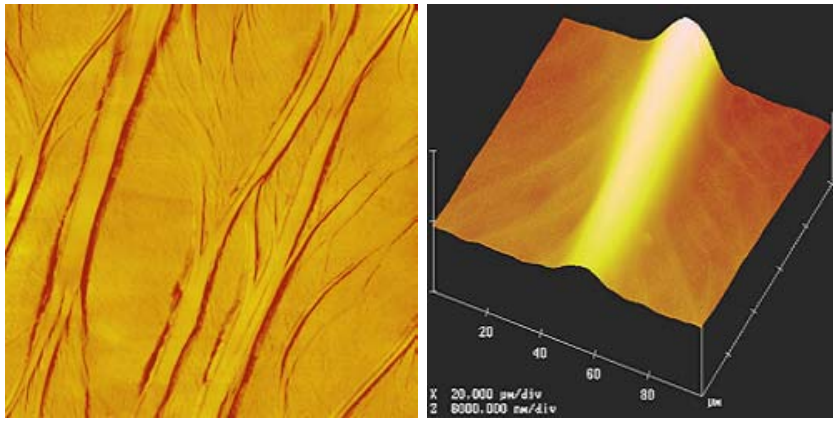

(b) Thick dendrite

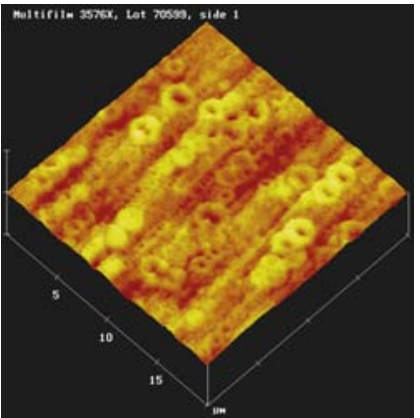

(c) Spherulites

Fig. 3. Crystallization introduced defects of iPP thin films.

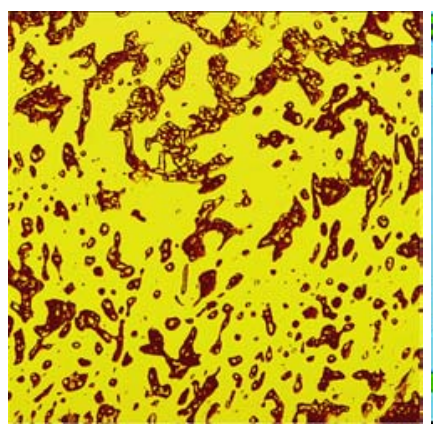

(a) Reactor $(50 \mu \mathrm{m})$

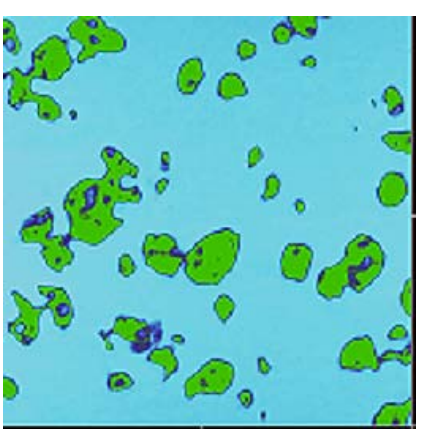

(b) Extruder $(50 \mu \mathrm{m})$

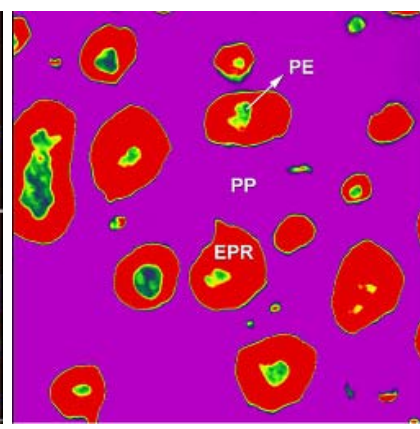

(c) 3 phases $(10 \mu \mathrm{m})$

Fig. 4. Phase images showing EPR particles in ICP.

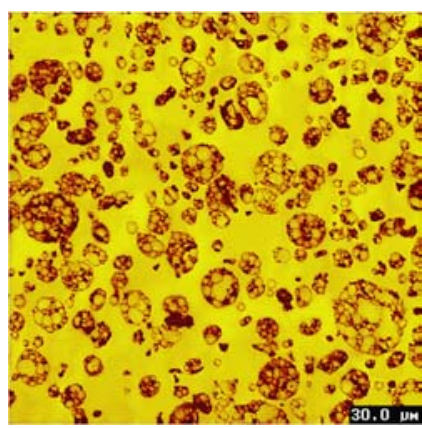

(a) $30 \times 30 \mu \mathrm{m}$

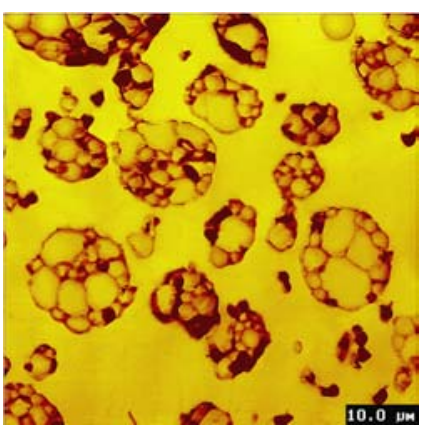

(b) $10 \times 10 \mu \mathrm{m}$

Fig. 5. Phase images showing rubber particles in HIPS.

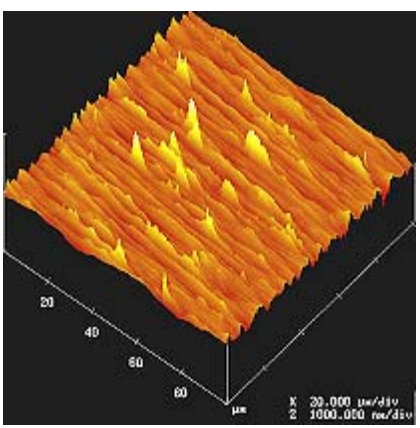

(a) Surface 3D

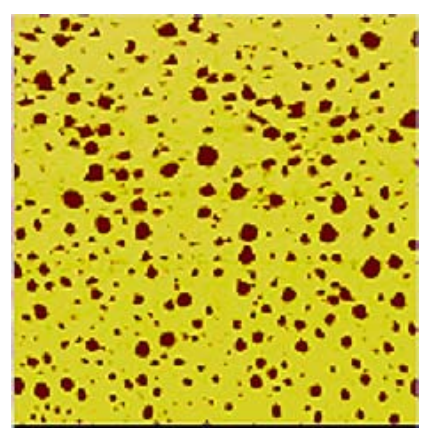

Fig.7. Phase image of $\mathrm{mPE}$ and $\mathrm{iPP}(20 \mu \mathrm{m})$.

Fig. 6. Height and phase images of a slit tape of iPP + EPR blend. 